\title{
The Design of the Internet of Things Solution for Food Supply Chain
}

\author{
Zhao Xiaorong $^{1, \mathrm{a}^{*}}$, Fan Honghui ${ }^{1, \mathrm{~b}}$, Zhu Hongjin $^{1,2, \mathrm{~b}}$, Fu Zhongjun $^{1, \mathrm{c}}$, Fu \\ Hanyu $^{1, d}$ \\ ${ }^{1}$ NO.1801, ZhongWu Avenue, Changzhou, China \\ ${ }^{2}$ Nanjing University of Science \& Technology, Nanjing China \\ a* zhaoxr432698@sina.com, ${ }^{\mathrm{b}}$ Fanhonghui@jsut.edu.cn. ${ }^{\mathrm{c}}$ june@jsut.edu.cn, , feiyu79@jsut.edu.cn
}

Keywords: Internet of Things; Survey; Machine to machine; Ubiquitous

\begin{abstract}
Many problems of foods safety had occurred in recent years, which resulted in public panic. As a result, the government has made food safety a top priority. In the last few years, the rapid development of the Internet of Things (IoT). How to make sure the security of food supply chain with the help of emerging technology - IoT, has the important practical significance. Iot is an inevitable choice to improve food logistics, and compare the content networking technology application before and after the food logistics management titer, thus must content networking technology application in food logistics, to food logistics management titer have larger stimulative effect. In this paper, we introduce a novel IoT architecture able to track and trace agriculture from the field through the supply chain and in food processing environments, realizing the food supply chain's effective information identification and traceability.
\end{abstract}

\section{Introduction}

In the last few years, we have seen an increased interest in the internet of things (IoT). Everyone's talking about IoT. Mostly, you hear how connecting sensor-enabled devices to the cloud can change everyday lives. That's true - there are some pretty cool connected consumer devices on the market. But the real value of the IoT lies in revolutionizing the way the world does business-and, in this case, the way food supply chains operate [1].

China has been plagued by tainted milk, pork, rice and other foods that have endangered people's health in recent years. Food and agricultural products from production to consumption of the whole process involves the production, processing, packing, transportation, storage, shelf display and consumption, each link is likely to bring the unsafe factors [2]. There is an imperative need for ensuring food quality across the supply chain. Achieving the objectives of food quality partly relies on physical traceability throughout the chain. As a result, the management of the supply chain, more significantly the cold chain related to the manufacture, distribution and sale of perishable, and condition-sensitive products, are seen as high priority applications [3, 4].

The application of IoT in food supply chains (FSCs) is one of the promising killer applications. Covering from precision agriculture, to food production, processing, storage, distribution, and consuming, so-called farm-to-plate, IoT solutions provide promising potentials to address the traceability, visibility and controllability challenges. Safer, more efficient, and sustainable FSCs are expectable in the near future [5]. 


\section{The IoT enabled food supply chain in action}

As shown in Table 1, definitions of IoT can be found in different researches, such as United States, European, Japan, and China. Although definitions from different organizations are somehow different, the requirements [6] for IoT are essentially the same, such as being able to integrate heterogeneous devices, ubiquitous data exchange, localization and tracking capabilities, and even being able to make the simple decision by themselves [7].

Table 1 The definitions and standards of IoT

\begin{tabular}{|l|l|}
\hline Organization Institute of & Web link \\
\hline $\begin{array}{l}\text { Massachusetts } \\
\text { Technology }\end{array}$ & http://www.autoidlabs.org/ \\
\hline EPCglobal & http://www.fas.org/irp/nic/disruptive \\
\hline National Intelligence Council & http://cordis.europa.eu/fp7/ict/enet/home_en.html \\
\hline European Commission & http://www.smart-systems-integration.org/public/internet-of-things \\
\hline European Commission & http://www.uidcenter.org/ \\
\hline Ubiquitous ID Center & http://www.iotcn.org.cn/ \\
\hline Internet of Things China &
\end{tabular}

The IoT makes possible a new cooperative between food producers, transportation and hospitality/retail companies who can work together as never before to ensure efficient delivery and food safety. With IoT-based business solutions, companies across the supply chain gain the real-time visibility and enable the automated, intelligent actions needed to ensure food is of the highest quality, delivered on time and prepared in optimal settings.

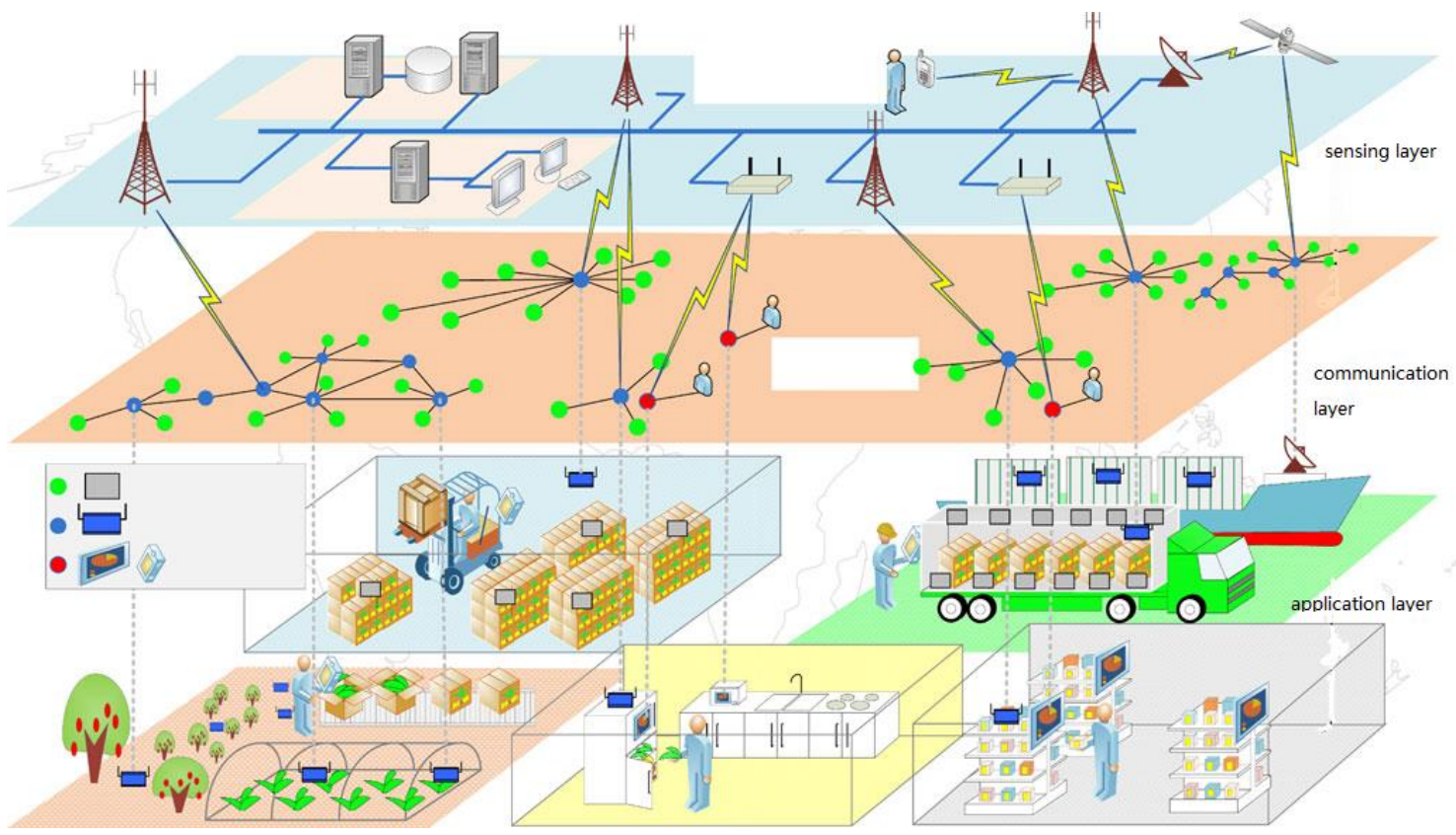

Figure. 1 A whole picture of food supply chains in the era of Internet-of-Things

For instance, food companies that use IoT-connected testing equipment can confirm food quality as it leaves the factory or warehouse. Fleet managers can then leverage the IoT to make sure 
temperature sensitive, perishable goods don't go bad in transit through sensor enabled refrigeration systems [8]. Any temperature fluctuations can trigger alerts that automatically adjust the truck's refrigeration. If the system is not able to auto-correct, an alert can be sent to the food supplier, who can replace bad goods before they arrive at the customer's dock. And by using an IoT-based fleet management solution to enable continual visibility into connected trucks, trucking managers can optimize routing and ensure on-time delivery of the new goods via an alternate vehicle. In the end, the customer receives quality goods on time and is never aware there was an issue. Plus, the sensor-enabled refrigeration system can send alerts to the manufacturer, pinpointing the exact part that broke down and facilitating faster replacement and fixes [9].

Today's typical FSC is a distributed system with large geographical and temporal scale, complex operation processes, and diverse technical requirements. It is impossible to map it into the virtual world without classification and formalization. We have abstracted the real FSCs into 5 scenarios: Produce, Store, Transport, Sell and Consume. A scenario is the abstraction of a class of similar deployment environments. It is not always equal to one transaction step in real business process; instead it may correspond to multiple transaction steps or a part of a single transaction step. Any real FSC can be composed by all or a part of the 5 scenarios under certain orders and topology.

As shown in Fig. 1, a typical IoT solution for a FSC comprises: a series of field devices (WSN nodes, RFID readers/tags, user interface terminals, etc.), a backbone system (databases, servers, and many kinds of terminals connected by distributed computer networks, etc.); and a series of heterogeneous wired and wireless communication infrastructures (WiFi, cellular, satellite, power line, Ethernet, etc). Due to its ubiquitous connectivity, all physical entities of field devices and backbone equipments can be distributed throughout the entire FSC. The vast amount of raw data is extracted and fused into high level and directly usable information for decision support systems (DSS).

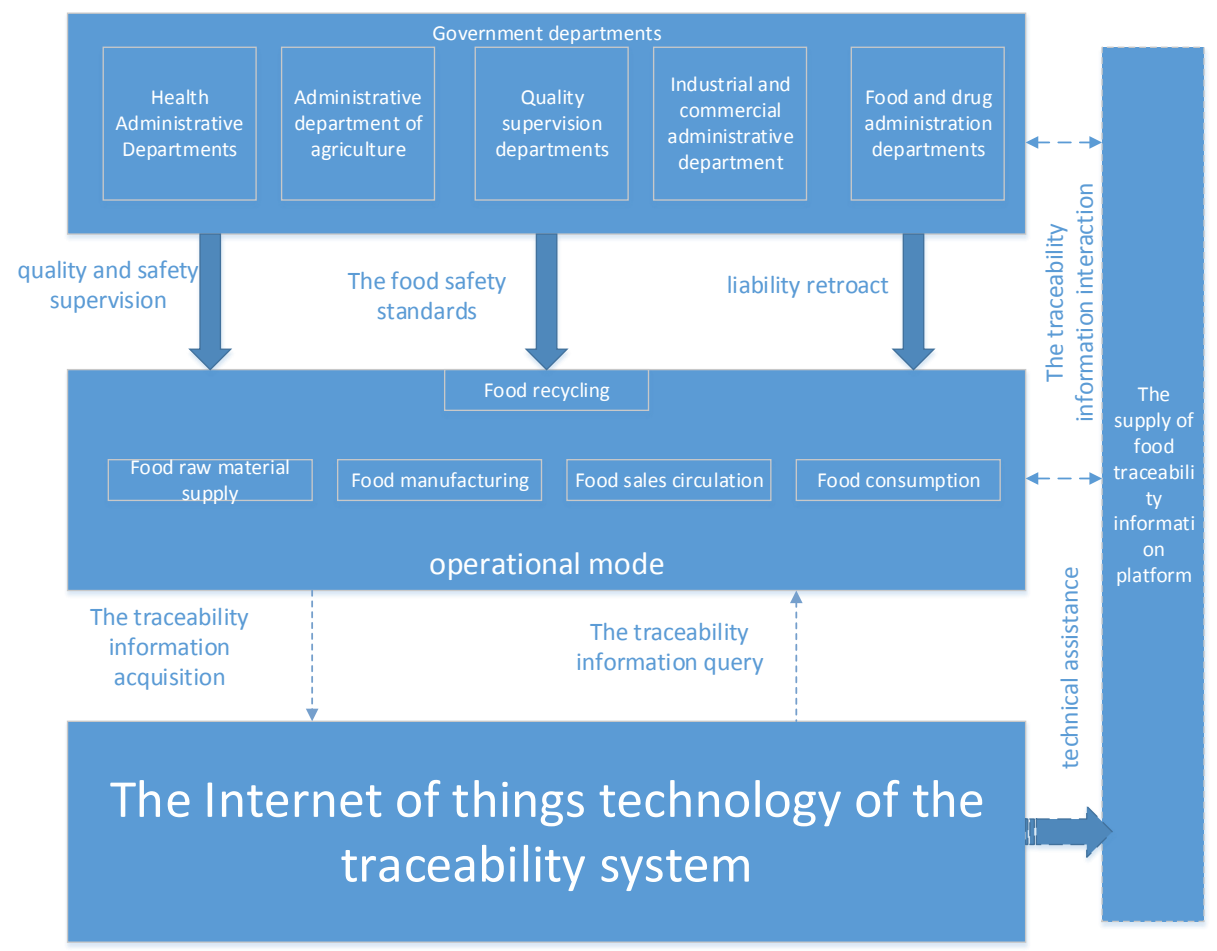

Figure. 2 The food supply chain traceability system model based on Internet of things

The food supply chain traceability system model can be seen in Fig.2. We have developed a platform that comprise three layers: sensing layer, communication layer and application layer. 
The sensing layer is designed to monitor the condition of crops and livestock on farms and in the supply chain with different automatic identification and data capture technologies, based on cost-effectiveness. RFID tags, for example, can be used to identify swine and cattle, as well as cases of high-value meats and fruits [10]. Cases of low-cost fruits can be tracked using 1-D or 2-D bar codes. Wireless sensor networks can monitor temperature, humidity, carbon dioxide, heavy metals and other environmental conditions in fields, greenhouses and housing for swine and cattle, as well as perishable items during transport.

The communication layer is designed to allow various stakeholders to access supply chain information. We set up an IoT architecture based on Object Name Service (ONS), so information can be captured and stored on the Web. Currently, the system tracks by lot level, but it will be able to manage goods at the item level using unique identifiers, such as a Serialized Global Trade Item Number or Global Individual Asset Identifier.

The application layer provides the functionalities that are built on top of an implementation of IoT, it will support applications and services that could be used by farmers, retailers, the government, analysts and consumers. It includes a database containing China's food safety regulations. Supply chain partners will be able to analyze data captured from the RFID tags and bar codes to determine product quality and shelf life. Farmers will be able to build their own applications and serviceswe've created some examples, including "my farm," "my crow house," "my supply chain," "tracking and tracing system" and "recall assistant. "Consumers will be able to check product expiration dates, quality guarantee periods, test reports, electronic pedigrees, product photos and videos, and customer evaluations.

The revolution of IoT technologies have brought out great potentials to make today's food supply chain safer, more effective and more sustainable.

\section{Summary}

In this paper, we introduce a novel IoT architecture able to track and trace agriculture from the field through the supply chain and in food processing environments. We proposed to use the IoT technologies to build the food logistics safety tracking system, analyzed the working principles and components of the system and through a series of calculation, obtained the positional, production, and safety information concerning the target food products.

\section{Acknowledgments}

This work was financially supported by National Natural Science Foundation (No.61302124), Scientific Research Foundation of Jiangsu University of Technology (No.KYY13030, KYY14020), the Natural Science Fund of Jiangsu Province (BK20130235), Natural Science Foundation of the Higher Education Institutions of Jiangsu Province (13KJB520006), Natural Science Fund of Changzhou (CJ20140049), Program of six talent tops of Jiangsu Province (DZXX-031), and the Key Laboratory of Cloud Computing \& Intelligent Information Processing of Changzhou City under Grant No.CM20123004. The supports are gratefully acknowledged.

\section{References}

[1] Jenifer Sunrise Winter: Ethics Inf Technol. Vol.16 (2014) No.1 p.27. 
[2] Liu Donghong, Zhou Jianwei, Mo Lingfei: Transactions of the Chinese Society for Agricultural Machinery. Vol.43 (2012) No.1, p.146. (in Chinese).

[3] Shen Junlong, Zeng Zhi: The Chinese Health Service Management. (2011) No.7, p.500. (in Chinese).

[4] Yao Yucheng: Jiangsu Agricultural Sciences. Vol.42 (2014) No.6, p.276. (in Chinese).

[5] Wang Mei: Logistics Technology. Vol.33 (2014) No.1, p.336. (in Chinese).

[6] Miorandi D., Sicari S., De Pellegrini F., \& Chlamtac I.: Ad Hoc Networks. Vol.10 (2012) No.7, p. 1497.

[7] Lopez T.S., Ranasignhe D.C., Patkai B., \& McFarlance D. C.: Information Systems Frontiers. Vol.13 (2011) No.2, p.281.

[8] Tomas Sanchez Lopez, Damith C. Ranasinghe, Mark Harrison, Duncan McFarlane: Pers Ubiquit Comput. Vol.16 (2012) No.3, p.291-308.

[9] Shang Peipei, Lin Guolong, Ma Zhanxin: Science and Technology of Food Industry. Vol.34 (2013) No.7, p.297. (in Chinese).

[10] Wang Jinpu, Wang Liang, An Introduction to the Internet of Things, PEKING UNIVERSITY PRESS, China, 2012, p.10. (in Chinese). 